\title{
Non-progressive cerebellar ataxia with intellectual disability
}

INSERM

\section{Source}

INSERM. (1999). Orphanet: an online rare disease and orphan drug data base. Nonprogressive cerebellar ataxia with intellectual disability. ORPHA:314647

Non-progressive cerebellar ataxia with intellectual deficit is a rare subtype of autosomal dominant cerebellar ataxia type 1 (ADCA type 1; see this term) characterized by the onset in infancy of cerebellar ataxia, neonatal hypotonia (in some), mild developmental delay and, in later life, intellectual disability. Less common features include dysarthria, dysmetria and dysmorphic facial features (long face, bulbous nose long philtrum, thick lower lip and pointed chin). 